tongue. There has aiso been a vesicle at the end of the penis, and there is now a crop of fresh-looking vesicles on the scrotum. The fluid from the vesicles is alkaline and clear. The red patches are becoming smaller and darker, and much resemble the fourth day of the rash of measles, so blotchy are they. On looking closely at what have been the larger red patches, they are disappearing in rings, exactly resembling the way that ringworm grows-that is, they become brownish-yellow in the centre, with a reddish ring encircling it.

30th. A few fresh vesicles on the chin and nose, which resemble in appearance the ordinary herpes from cold, but no vesicles at all on the lips. Has severe pain again in abdomen.

Nov. 3rd-Skin desquamating all over, more or less, in large and small scales. The patches with yellowish centre and red ring surrounding are very marked, but they are all manner of shapes and sizes. Fainted while at night-stool; slept much after.

6th.-Skin still peeling; convalescent

His treatment was salines at first, with Dover's powder to relieve the pain; subsequently he had the mineral acids with bark, and then quinine. This illness, together with the mitral disease, was the cause of a very great debility, extending over some months.

\section{CASE OF CLEFT HARD AND SOFT PALATE, OPERATED ON BY LANGENBECK'S METHOD ; RESULT PERFECT.}

BY GEORGE BUCHANAN, M.A., M.D., PROFESSOR OF CLINICAT SURGERY, UNIVRASITY OF GLASGOW.

DAvid $\mathrm{S}-$, aged twenty-two, was admitted to the Western Infirmary Nov. 26th, 1875. There was a congenital cleft in the palate involving the whole of the soft and half of the hard palate. The anterior end of the fissure was quite rounded, and the margins of cleft in the hard palate were three-quarters of an inch apart. The tips of the bifid uvula were drawn asunder by the palatine muscles.

The operation was performed after the method introduced by Langenbeck, closure of the hard and soft palate being accomplished simultaneously. The patient, not being put under the influence of chloroform, was able to give great assistance, by holding his head at a proper angle and occasionally stopping to rest and wash his mouth. From the help of the patient the proceedings were much more rapid and satisfactory than usual.

I first carefully made raw the entire edges of the gap by cutting off a thin slice of tissue from the hard palate, to the tips of the uvula, on both sides, taking care that the raw edge was continuous throughout. I then made an incision down to the bone, in the roof of the mouth, close within the alveolar process, extending from the hamular process forward to near the incisive foramen. Then with a blunt periosteal elevator of a hooked form I stripped the sof tissues, including the periosteum, from the palate processes of the maxillary and palate bones, and the flap so separated hung down like a curtain from the roof of the mouth, but of course supported by its attachment in front and behind. This was done on each side. The posterior part of the cleft, however, still remained too far apart to allow of approximation without considerable tension, so I relieved this by making incisions through the soft palate on each side, as in Dieffenbach's operation. The edges of the cleft could now be drawn together throughout the whole extent. Six silk threads were introduced by the ingenious method introduced by Sir William Fergusson, and finally by these, fine silver wires were drawn through and tied. At the end of the operation the gap was seen to be closed throughout in a most satisfactory manner.

On the eighth day the silver sutures were removed, and the edges found to be firmly adherent.

The patient was kept in the hospital for some time longer in order to see that the granulation of the side wounds should not reopen the cleft.

Two months after the operation the palate was firmly closed throughout its whole extent. Already patient felt the difference in swallowing, and the improvement in his articulation was manifest. In fact, when be paid proper attention he could speak in such a way that his former deficiency was scarcely recognisable, and there is no doubt that by a little practice he will still further improve.

The operation, so far as regards the union of the cleft, and the result, both on swallowing and speech, is the most successful in my experience, and leaves little to be desired. Glasgow.

ON

\section{THE ORIGIN OF INFANTILE SYPHILIS.}

BY ROBERT CORY, M.B.,

ASSTSTANT OBSTETRIC PHYSICIAY TO ST. THOMAS'S HOSPITAT.

IN The Liancet of March 25th, I saw, by the report of Dr. Greenfield's speech at the Pathological Society, that he had generously mentioned $\mathrm{my}$ name in reference to a sug. gestion $I$ had made to him in conversation upon infantile syphilis. I should not at present have ventured to give greater publicity to this theory if my name had not been mentioned in connexion with it, because for substantiation it requires more accurately recorded facts than $I$ am as jet able to bring forward.

When infantile syphilis was first recognised, it was very naturally attributed to direcl inoculation at the time of birth. Afterwards, however, when it was found that, except in a few instances, the mother was not suffering from local sores at the time of birth, another explanation was offered, which is still very generally heldnamel $y$, that the disease was inherited like other hereditary diseases, and that consequently it was communicated to the offspring at the time of conception. A corollary followed from this-namely, that the diseased fœtus might indirectly convey to the mother the disease of the father. Now, looking at the facts and arguments in support of these views, they seem only partially to support it.

Let us consider the cases adduced first. They may be divided into two classes: - 1st. Those where, the father having had syphilis, the mother gives birth to a child which afterwards suffers from the disease, but she herself remains absolutely free. 2ad. Cases where the mother, during pregnancy, without any discovered primary inoculation, suffers from syphilitic symptoms. In some of these cases the woman is said to suffer during each pregnancy, but remains well in the interval.

The alleged cases of the first class are the most im portant, for, if true, they are conclusively in favour of the received theory. M. Diday mentions several of these in his work on "Infantile Syphilis," but one whic's he details is sufficient to throw suspicion on all, for it indicates how a fallacy may arise, and also shows how facts may be bent to theories. The case is this. "The wife of a syphilitic man gave birth to a child which became syphilitic. The woman, however, suffered from no symptom whatever, unless a small patch of granular redness on the fore part of the neck could be said to be such. This woman becoming a widow, married a bealthy man, but again by him gave birth to a syphilitic child." M. Diday supposes in this case that an impression was left behind on the woman by her first husband, whi:h was capable of infecting her subsequent children, in some such way. I suppose, as the subsequent foals of a mare once covered by a zebra are sometimes impressed with the markings of the zebra. He does not, however, admit that she had herself contracted syphilis, because she presented no symptoms, and he argues against the small patch of redness being such. Here he thinks that it was possible for a woman to be free from syphilis herself, yet to give birth to a syphilitic child, the result of a former husband's impression, the real father being healthy. This assumption is directly contrary to the fact observed and stated by Mr. Hutchinson, "that he has never known a motber infested by suckling her syphilitic child;" evidently because she is protected by a previous attack of the disease. We see, therefore, of how little value the first class of cases become. 
With regard to the second class of cases, I think an explanation can be found more conformable to facts than the present interpretation, and it is this: That in all these, where the mother shows symptoms of syphilis while carrying her child, and afterwards gives birth to an apparently healthy child, which is subsequently attacked, she infects her offspring at birth by her blood; for we can readily suppose that, at the time of the separation of the pla. centa, before the foetal circulation has ceased, some of the mother's blood may be swept into the fotal circulation along the umbilical vein. If this be so it will also explain why the liver is so early and seriously affected in infantile syphilis. The only difficulty left is the question-How is the mother affected if not by the foetus? In answer to this I will offer a suggestion-namely, that previously to her pregnancy the muther has been infected by an ovum impregnated with a diseased spermatozoon, and the necessary result of this has been a very early abortion, so early perhaps as only to cause a retarded menstruation; that nltimately two healthy elements have combined, with the result of producing a healthy fœtus. In some cases, moreover, a primary inoculation may have been overlooked. It really seems quite incredible that a child, the offspring of a diseased first element, should apparently be in perfect health at birth, and moreover should have transmitted, long before this time, a disease to its parent of which it shows no symptoms itself. Another fact which supports these views is the period elapsing between the birth and the manifestation of the disease, which, though perhaps a little shorter than the incubatory period of syphilis, is yet not far from coinciding.

Another difficulty with the present received views is the great difference between syphilis and the other hereditary diseases. If it be such it stands forward as a marvellous exception to the others, for not one of them has been proved to be contagious or inoculable, nor does one attack of any of them convey an immunity against another attack. In most, if not all, the individuals subject to hereditary disease health remains until they are subjected to the noxious influences which gave rise to the disease in the parents. In other words, they are born with a greater liability to be influenced by external irritants. Some portion of their construction is weak, but they are not born with the disease.

This is not what is contended for syphilis. Here a disease is fostered in the growing embryo; it is built up with it, but it is not destined to show itself until three or four weeks after birth. No care can avoid the attack. The disease is present, is strictly inherited. The difference therefore is easily seen, because if syphilis corresponded to the other hereditary diseases, a child would be born with a greater liability to take syphilis, but not with the disease. There are some individuals who are born with a liability to take small-pox. These may have it three or four times in their life. They inberit small-pox in the same way as another inherits phthisis; for I am one of those who believe phthisis is always the result of external irritants acting upon a weak lung; the weak lung is inherited, but not the phthisis.

We know that structural differences are inherited, and it is probable that all so-called hereditary diseases depend on structural peculiarities.

Lastly, if we glance at the explanation that has been offered to account for the manifestation of syphilis in the infant four weeks or so after birth, I think we must confess that it leaves the matter in as great obscurity as ever. I refer to the suggestion of Mr. Hutchinson-viz., that the child may manifest symptoms of syphilis when exposed to the light in some such way as chlorophyl is developed in the leaves of a plant, or as the tadpole is developed in to the frog by the same influence.

I will sum up in a few words the proposition I have tried to maintain. First, that in most cases of infantile syphilis the foetus remains healthy until the time of the separation of the placenta, that it is then infected by the blood of the mother, and therefore that in all such cases the mother must have been primarily infected. Secondly, that it is not improbable that the muther is first infected by an early abortion, so early perhaps as only to cause a retarded menstruation, or that in some cases she may have had an overlooked primary sore.

Palace-road, S.E.

\section{Ittedical Societies.}

\section{ROYAL MEDICAL AND CHIRURGICAL SOCIETY.}

THE last meeting of this Society, held during the present session, took place on the 23rd ult., the chair being occupied by Dr. Ed. Ballard, one of the Vice-Presidents.

The first paper read was by Dr. W. H. BroadBENT on "Ingravescent Apoplexy, a contribution to the Localisation of Cerebral Lesions." The following is an abstract of the paper:-Sir Thomas Watson, following Abercrombie, de scribes three forms of apoplectic attack. The first sudden, with loss of consciousness and stertorous breathing; the second beginning with sudden pain in the head, faintness, and romiting, followed by gradually increasing coma; the third characterised by sudden hemiplegia without loss of consciousness. To the second of these modes of attack the term "ingravescent" has been applied; its characteristic features are absence of loss of consciousness at the outset, gradual accession of symptoms, and speedily fatal termina. tion; and the cause is invariably found to be a large extravasation of blood. The object of this communication is to show that not only is the hæmorrhage large in these cases, but that its situation is, within certain limits, constant, and also that an anatomical explanation can be given of the phenomena of the attack. The seat of hæmorrbage is on the outer side of the extra-ventricular corpus striatum ("lenticular nucleus" of foreign writers), between this ganglion and the "external capsule"; and the explanation offered is as follows:-That the large amount of blood extravasated arises from the large size of the vessels here found, and from the slight resistance opposed to the effusion, and that the absence of loss of consciousness is due to the fact that there is little laceration of fibre or com= pression of the brain. The situation and relations of the clot are best seen by opening out the fissure of Sylvius, when the convolutions of the island of Reil will be found to be obliterated, and spread out over the effused blood. Afterwards by the usual sections the further course of the extravasation into the ventricle or into the substance of the hemisphere can be traced. The following cases are related:-CASE 1. A man, aged fifty, was suddenly seized with giddiness and slight mental confusion; left hemiplegia was early noticed, but there was no loss of consciousness. He was brought immediately to St. Mary's Hospital, and on admission, though a little excited, he gave an accurate account of the circumstances attending the attack. There was left hemiplegia, with relaxation of the paralysed limbs no distortion of face, but extreme conjugate deviation to the right of the head and eyes; marked hemianæsthesia affecting face and body, as well as limbs; slight reflex action on tickling left sole. Pulse 108, long and firm, but weak (?) A tendency to sleep became manifest, and the nature of the case being recognised, an attempt was made to bleed. Three veins were opened in the arms, but only a trifling amount of blood could be obtained; the jugulars could not be made to fill sufficiently. Vomiting interrupted further proceedings. The patient continued to sleep, but could be roused till about eight hours after the attack, when stertor suddenly set in ; he was then found to be quite unconscious, and in about twenty minutes be died. During the final stertor the lateral deviation of the ejes ceased; the heart was felt to beat for seven and a half minutes after cessation of respiration. On post-mortem examination, hæmorrhage of large amount was found to have taken place between the corpus striatum and external capsule, flattening out and distending the convolutions of the island of Reil, forming a cavity along the outer side of the corpus striatum and thalamus two and a half or three inches long, and penetrating the lateral ventricle by a fissure extending almost the entire length of its onter angle. All the ventricles were filled with blood. There were bæmorrhages into the lungs, spleen, and liver; kidneys contracted and granular.-CASE 2. A woman, aged forty.five, had slight paralysis on January 8th, 1871, which speedily passed 